\title{
Estudios experimentales de la biomecánica de la marcha durante el embarazo: Revisión sistemática de ensayos clínicos (2000-2018)
}

Eva María Martínez Jiménez', José Ignacio Díaz Velázquez², Rubén Sánchez Gómez³, Fernando Santiago Nuño ${ }^{4}$, Israel Casado Hernández ${ }^{5}$ Xavier Garrido Castells ${ }^{6}$

Fecha de recepción: 4 de junio de 2018 / Fecha de aceptación: 12 de diciembre de 2018

Resumen. Background: El embarazo es un momento en la vida de la mujer cuyos cambios fisiológicos, hormonales, y morfológicos generan grandes cambios corporales. Estos cambios se producen en un periodo de tiempo muy corto y provocan una pérdida de estabilidad y de cambios cinemáticos y cinéticos en su marcha que generan caídas y molestias musculoesqueléticas en el miembro inferior y pie.

Métodos: Se ha realizado una revisión de la literatura científica para identificar los posibles estudios científicos sobre la marcha y embarazo. Se han consultado los principales buscadores y bases de datos biomédicas: Medline-pubmed, Enfispo, Scielo y Science Direct ${ }^{\circledR}$. El periodo de búsqueda estuvo comprendido febrero-junio de 2018.

Resultados: En nuestra búsqueda hallamos 912 resúmenes de artículos con las palabras clave seleccionadas. 892 artículos de los anteriormente mencionados fueron excluidos tras leerlos por no cumplir los criterios de selección del estudio. Por lo tanto, obtuvimos 20 relevantes.

Conclusión: Existen estudios experimentales que evidencian cambios en la cinemática, cinética de la marcha del tronco, pelvis, miembro inferior y pie en el embarazo y postparto.

Palabras clave: marcha; embarazo; cinemática; cinética; extremidad inferior.

\section{[en] Experimental studies of gait biomechanics during pregnancy: Systematic review of} clinical trials. (2000-2018)

Abstract. Background: Pregnancy is a moment in a woman's life whose physiological, hormonal, and morphological changes generate great bodily changes. These changes occur in a very short period of time and cause a loss of stability and a series of kinematic and kinetic changes in their gait that cause falls and musculoskeletal discomfort in the lower limb and foot.

Methods: A review of the scientific literature has been carried out to identify possible scientific studies on gait and pregnancy. The main biomedical search engines and databases were consulted: Medline-Pubmed, Enfispo, Scielo and Science Direct ${ }^{\circledR}$. The search period was comprised February-June 2018.

Results: In our search we found 912 summaries of articles with the selected keywords. 892 articles of the aforementioned were excluded after reading them because they did not meet the selection criteria of the study. Therefore, we obtained 20 relevant.

Conclusion: There are experimental studies that show changes in the kinematics, kinetics of the trunk, pelvis, lower limb and foot in pregnancy and postpartum.

PhD Profesora de Postgrado en la Universidad Rey Juan Carlos. Diplomado en Fisioterapia y Podología.

Adv. Castilla La Mancha local 47. 28702 S. S. de los Reyes. Madrid

eva.hache2@hotmail.com

2 Diplomado en Fisioterapia y Podología por la Universidad Complutense de Madrid.

josemarcha@hotmail.com

3 PhD. Profesor del Departamento de Enfermería. Facultad de Enfermería Fisioterapia, Podología de la Universidad Complutense de Madrid. España.

rusanc02@ucm.es

4 Fisioterapeuta y Podólogo por la Universidad San Pablo CEU. Doctorando en la Universidad de A Coruña. fersanti@hotmail.com

$5 \quad$ PhD Grado en Podología Universidad Complutense de Madrid.

israelcasado@yahoo.es

6 Doctorando en la Universidad da Coruña. Diplomado en Podología por la Universitat de Barcelona

xavi3garrido@hotmail.com 
Keywords: gait; pregnancy; kinematics; kinetics; lower extremity.

Sumario: 1. Background.2. Métodos. 3. Resultados. 3.1. Variables espaciotemporales. 3.2. Cinética. 3.3. Cinemática. 4. Discusión. 5. Conclusiones. 6. Bibliografía.

Cómo citar: Martínez Jiménez, E. M. et ali (2019) Estudios experimentales de la biomecánica de la marcha durante el embarazo: Revisión sistemática de ensayos clínicos (2000-2018), en Revista internacional de ciencias podológicas 13(1), 77-86.

Los autores declaran no tener ningún tipo de interés económico o comercial.

\section{Background}

El embarazo se caracteriza por grandes cambios producidos en la mujer, condicionada por variaciones psico-emocionales, fisiológicas y morfológicas ${ }^{1 .}$.

Los cambios musculoesqueléticos se ven producidos por diferentes factores, entre los cuales se consideran como más relevantes el aumento de peso, el cambio hormonal y el cambio postural que genera el aumento de volumen y peso del feto ${ }^{2}$.

El aumento de peso durante el embarazo está causado por: el aumento de volumen sanguíneo, el aumento del volumen del pecho y el aumento de grasa y fluido extracelular ${ }^{3}$. Un aumento de peso normal o ideal se considera en base al BMI anterior al embarazo. Mujeres con un $\mathrm{BMI}<18.5$ aumentan su peso entre $12.5-\mathrm{Kg}$ a $18 \mathrm{Kg}$, con un BMI de entre 18.5 a 25 (normopeso) aumentan de 11.5 a $16 \mathrm{Kg}$. Las mujeres con sobrepeso previo (BMI entre 25 y 30 ) aumentan su peso de 7 a $11.5 \mathrm{Kg}$. Las mujeres obesas aumentarían su peso de $5 \mathrm{a} 9 \mathrm{Kg}$ idealmente ${ }^{4}$. Una de cada 5 mujeres es obesa al quedarse embarazada ${ }^{5}$ aumentando el riesgo de todas las complicaciones para la mujer y para el feto durante el embarazo, incluidas las musculoesqueléticas 5 .

Dentro del cambio hormonal que sufre la mujer embarazada la relaxina parece tener un papel determinante en la biomecánica durante el embarazo ${ }^{6}$. La relaxina genera una mayor laxitud ligamentosa en la pelvis $\mathrm{y}$ en las articulaciones periféricas ${ }^{7,8}$. Y tienen una relación directa con el nivel de relaxina en sangre cuyo pico máximo se produce a la duodécima semana de gestación ${ }^{6}$, preparando así osteoarticularmente el cuerpo de la embarazada al parto y a los cambios morfológicos. Esta laxitud es mayor en el segundo embarazo y todavía mayor en el tercer embarazo respecto al segundo. Posteriormente al parto los ligamentos permanecen con una mayor laxitud durante 6 meses ${ }^{7,9}$.

El aumento del peso del feto genera cambios adaptativos en la columna lumbar ${ }^{2}$, que genera lumbalgias en más de un $50 \%$ de las mujeres embarazadas ${ }^{2}$. La lordosis lumbar estabiliza la parte superior del cuerpo sobre las extremidades inferiores, colocando el centro de gravedad sobre las caderas y la base de sustentación ${ }^{10,11}$. Estudios en homínidos revelan que duranteel embarazo se producen dos fases. En la primera el centro de gravedad se desplaza anteriormente hasta que el feto alcanza un $40 \%$ de su peso esperado ${ }^{10}$. En una segunda fase, cuando el peso del feto aumenta más de $40 \%$, la mujer aumenta la lordosis lumbar para mantener el control del centro de gravedad $^{10}$. En humanos, algunos estudios han encontrado una elevación y anteriorización del centro de gravedad ${ }^{2}, 12$, mientras otros observan un desplazamiento superior y posterior ${ }^{13}$. Las perturbaciones del centro de gravedad, junto con el resto de cambios posturales y biomecánicos conducen a un cambio de la postura y equilibrio ${ }^{14,15}$.

A pesar de estas modificaciones el $27 \%$ de las embarazadas sufren caídas durante el embarazo, y con un coste biomecánico importante. Estudios en 3 dimensiones indican que durante el embarazo hay una mayor demanda en los músculos abductores y extensores de cadera y en los músculos flexores plantares durante la marcha ${ }^{12}$, las molestias músculo esqueléticas constantes en columna y miembro inferior también pueden generar ajustes sistemáticos de postura estática y dinámica que ocasionen alteraciones en el desarrollo de la marcha ${ }^{12}$.

El cambio del centro de gravedad, el comportamiento mecánico de los tejidos, la disminución del nivel de actividad física y 
las ganancias de peso corporal condicionan la funcionalidad de esta población ${ }^{12}$. Por ello, el profesional sanitario debe recomendar realizar actividad física regular durante el embarazo, de grandes grupos musculares de manera continua y rítmica como caminar ${ }^{6}$. Aunque no todos los cambios biomecánicos durante el embarazo son conocidos, las adaptaciones y grandes solicitaciones del miembro inferior hacen esencial el papel del podólogo en esta población diagnosticandola y tratandola biomecánicamente.

\section{Métodos}

Se realizó una revisión de la literatura científica para identificar los posibles estudios experimentales sobre la cinemática y la cinética de la marcha durante el embarazo. Se consultaron los principales buscadores y bases de datos biomédicas: Medline-pubmed, Enfispo, Scielo y Science Direct ${ }^{\circledR}$. El periodo de búsqueda estuvo comprendido febrerojunio de 2018. Las palabras clave fueron "gait" con el operador boleano OR "walk" además del operador bolenao AND "pregnacy". Los criterios de inclusión y exclusión se muestran en la tabla 1.

Tabla 1. Criterios de inclusión y exclusión.

\begin{tabular}{|l|l|}
\hline Criterios de inclusión & Criterios de exclusión \\
\hline Publicación entre 2000- febrero 2018 & Publicación anterior al año 2000 \\
\hline Embarazadas sanas & Embarazadas con historia de dolor \\
\hline Mujeres entre 20 y 40 años & Revisiones \\
\hline Estudios experimentales. & $\begin{array}{l}\text { Estudios observacionales de variables no } \\
\text { cuantitativas. }\end{array}$ \\
\hline $\begin{array}{l}\text { Estudios con variables cuantitativas, con } \\
\text { sistema de medición optoeléctricos de imagen, } \\
\text { plataformas, 3 dimensiones u otros }\end{array}$ & \\
\hline
\end{tabular}

\section{Resultados}

En nuestra búsqueda hallamos 912 resúmenes de artículos con las palabras clave seleccionadas. De ellos 892 artículos de los anteriormente mencionados fueron excluidos tras leerlos por no cumplir los criterios de selección del estudio. Por lo tanto, obtuvimos 20 relevantes, En la Figura 1 se puede observar el resultado de la búsqueda. 


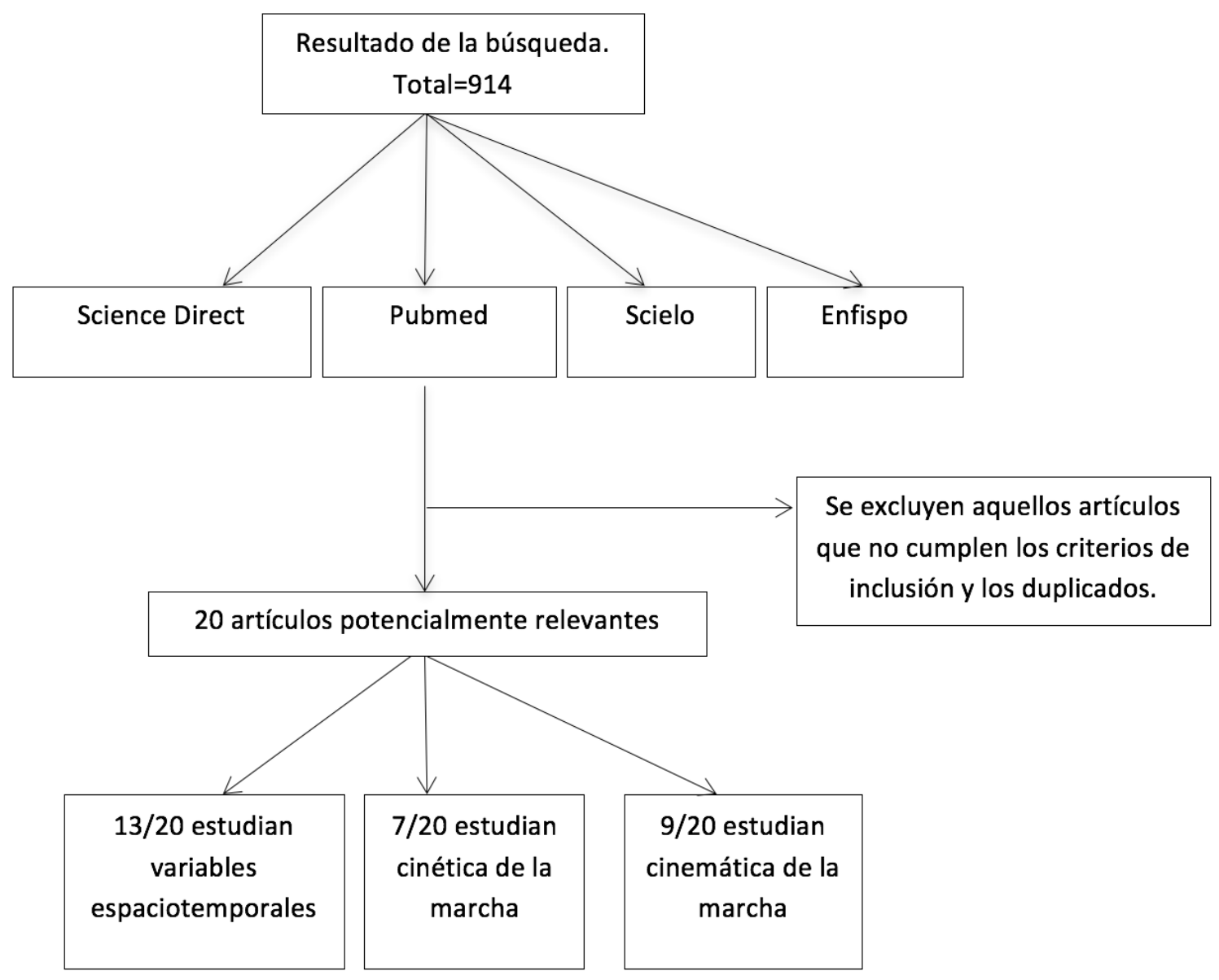

Figura 1. Flujograma de la búsqueda

\subsection{Variables espaciotemporales}

En el estudio de las variables denominadas en la tabla 1 como espaciotemporales y de distancia. La mayoría de los estudios encuentran: una disminución en la velocidad de la marcha ${ }^{15}$ (especialmente en el tercer trimestre), disminución en la longitud del paso $^{15,16,17}$ (progresiva a medida que avanza el embarazo) y aumenta la anchura del paso (progresivamente también con la evolución) $11,15,20,21,22$ y un aumento de la base de sustentación ${ }^{17} \mathrm{t}$

La duración de las fases de la marcha también se ven alteradas. Se produce un aumento temporal de la fase de doble apoyo de la marcha ${ }^{12,15,16,19,23}$ aproximadamente de un 6 a un $12 \%$ en los 4 últimos meses de embarazo ${ }^{24}$ y una disminución de la fase de apoyo unipodal de la marcha ${ }^{12,19,23}$ y en la longitud del ciclo de marcha $^{16,25,19,21}$.

La duración de cada subfase de la fase de apoyo de la marcha(contacto de talón, fase media de apoyo y fase de despegue) también es mayor en el segundo y tercer trimestres respecto al postparto ${ }^{26}$. Tras el parto la velocidad se recupera inmediatamente en un $3 \%^{24}$ y progresivamente vuelve a la normalidad, así como las adaptaciones espaciotemporales de la marcha, tras los 6 meses posteriores al parto ${ }^{15}$.

\subsection{Cinética}

En el estudio de las fuerzas que se producen durante la marcha ${ }^{27}$, fuerzas de frenado $\mathrm{y}$ empuje resistencia a la prono-supinación, momentos de fuerzas y potencias de trabajo a partir de cada zancada ${ }^{12,28}$ encontramos distintos hallazgos.

Durante el embarazo se modifican las fuerzas de inercia del tronco inferior en la marcha y al subir escaleras, especialmente al final de la gestación ${ }^{29}$ Además, se han encontrado evidencias en aumento del momento extensor ${ }^{30}$ así como de los abductores de la cadera ${ }^{12}$, una 
disminución en el momento extensor de la rodilla $^{30}$ y disminución del momento adductor de la rodilla ${ }^{30}$, una disminución también en el momento plantarflexor de tobillo ${ }^{30,12}$. Hay autores que relacionan estos cambios y también el aumento en la anchura del paso y la reducción de la inclinación pélvica ${ }^{11}$ con la reducción del desplazamiento del centro de gravedad durante la marcha a medida que progresa el embarazo ${ }^{31}$. Concretamente, la amplitud de desplazamiento mediolateral se reduce un $20 \%$, la anteroposterior un $16 \%$ y la superoinferior un $5 \%$, así como la velocidad a la que es desplazado el Centro de gravedad ${ }^{16,31}$. Este hecho se ha evidenciado al inicio de la marcha en las gestantes ${ }^{31}$, y en la marcha establecida ${ }^{11,24}$.

Estudios que registran la línea de marcha (que describe el centro de presiones sobre la huella plantar) demuestran cambios en la velocidad del centro de presiones respecto a un pie normal ${ }^{26}$. Además, la línea de marcha cambia en el segundo trimestre produciéndose una desviación interna respecto al postparto al final de la fase de contacto de talón e inicio de la fase media de apoyo. En el tercer trimestre produce una desviación externa de la línea de marcha en la fase final de la fase de contacto de talón a inicio de la fase media de apoyo comparado con el postparto ${ }^{26}$. En el despegue se produce en ambos trimestres una desviación lateral de la línea de marcha, mayor en el tercer trimestre ${ }^{26}$.

\subsection{Cinemática}

Es el estudio de la dinámica del movimiento de la marcha a través de sus variaciones angulares y desplazamientos de los diferentes segmentos corporales ${ }^{12}$ Los autores de estos estudios encuentran los mismos cambios cinemáticos entre trimestres, pero con una influencia clara con el peso relativo ganado, más que el peso neto en sí de la embarazada ${ }^{11}$. Estos estudios revelan que existe una reducción de la velocidad de la marcha ${ }^{17,18,32}$ y reducción de la cadencia ${ }^{32,33}$. En los últimos cuatro meses de embarazo la velocidad disminuye un $22 \%{ }^{24}$

La cinemática del miembro inferior demuestra cambios significativos principalmente en la pelvis y la cadera, que comienzan por un movimiento mayor de anteversión de la pelvis durante la fase de apoyo de la marcha ${ }^{26,34}$. Se estima de media que aumenta en 5 grados $^{12}$, aunque se incrementa progresivamente según avanza el embarazo por el aumento de crecimiento del feto y el abdomen ${ }^{26}$.

En el plano sagital se produce un aumento de la flexión de cadera de en la fase de apoyo de la marcha debido a la posición de anterversión de la pelvis ${ }^{16,18,23}$ pero el rango articular de la cadera en flexión y extensión utilizado durante la marcha se ve reducido como consecuencia de la reducción de la longitud del paso. El incremento en de la rotación externa y la reducción de la separación de cadera está relacionado con una mayor anchura del paso $^{23}$. La flexión de rodilla ve aumentado su rango en la parte final de la pase de apoyo de la marcha ${ }^{19}$ y en esta fase final también disminuye la flexión dorsal de tobillo ${ }^{18}$. La flexión plantar se reduce en la fase despegue ${ }^{18}$ y se disminuye la eversión del tobillo, especialmente en el tercer trimestre ${ }^{26}$. Esto ha sido relacionado por algunos autores con una estrategia para mejorar el control postural ante un tobillo más rígido durante el embarazo y aportar estabilidad a la marcha $^{26}$.

La pelvis además en su plano frontal se inclina menos lateralmente durante la fase de apoyo $^{1134}$, lo que implica una disminución de la inclinación lateral y rotación de la columna lumbar ${ }^{34}$

Estudios sobre el tórax durante la marcha muestran una traslación lateral de las vértebras cervical $7^{\circ}(\mathrm{C} 7)$ y lumbar $4^{\circ}(\mathrm{L} 4)$, así como una mayor extensión torácica en el último trimestre, un menor rango de movimiento en flexión de la columna torácica en el tercer trimestre respecto al segundo ${ }^{22,34}$, que se consideran adaptaciones para aumentar el volumen abdominal ${ }^{22}$. 


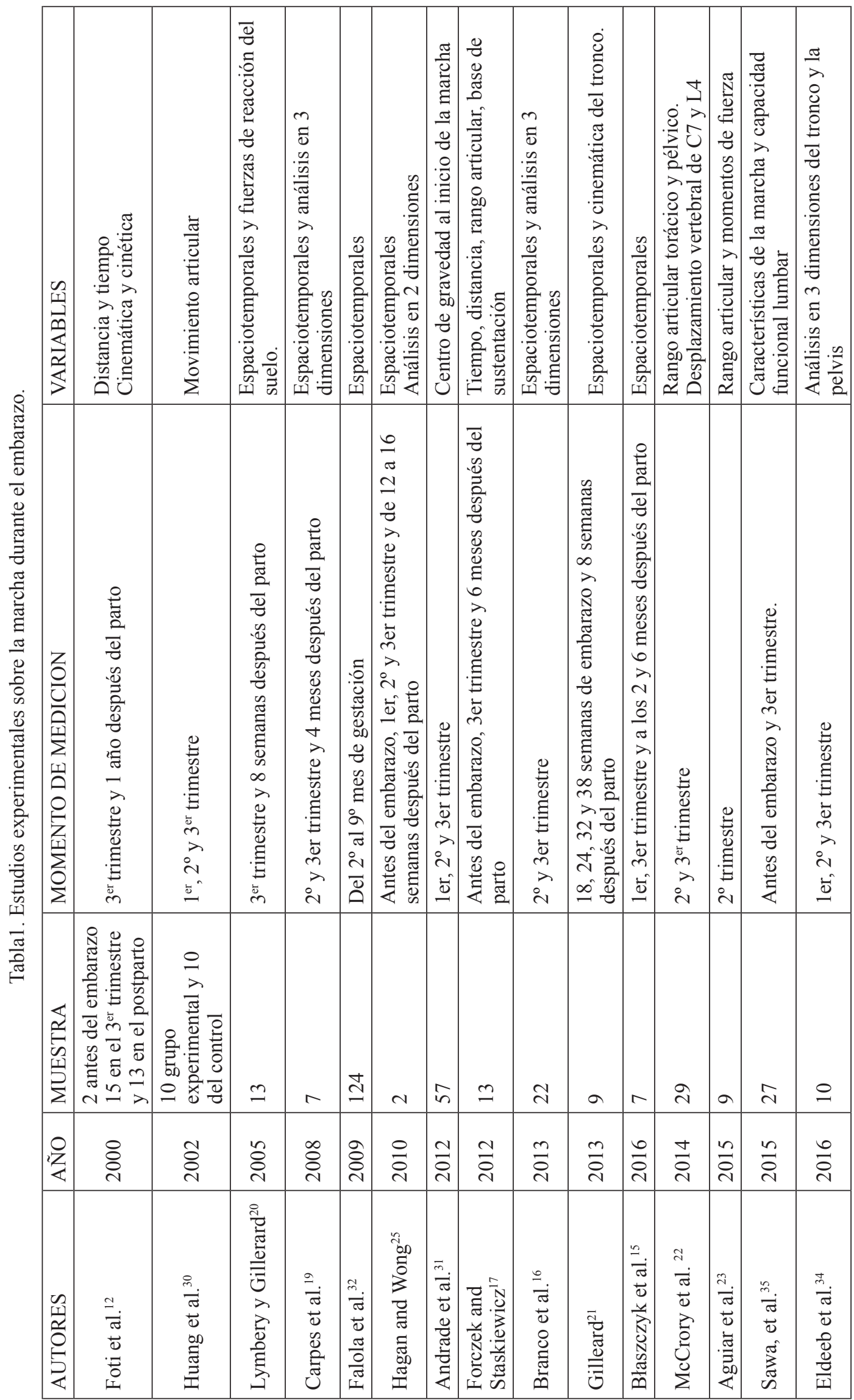




\begin{tabular}{|c|c|c|c|c|}
\hline 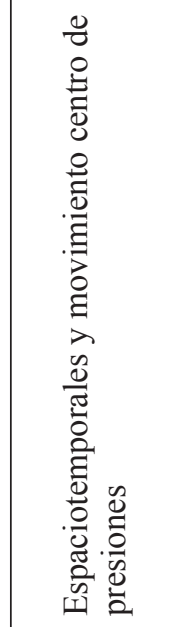 & 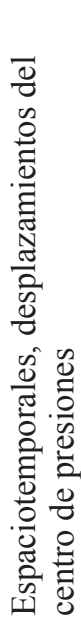 & 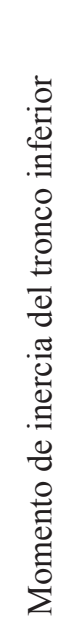 & 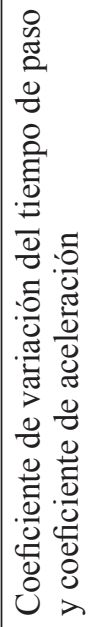 & 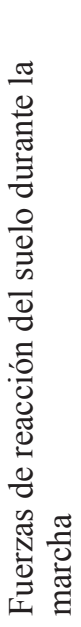 \\
\hline 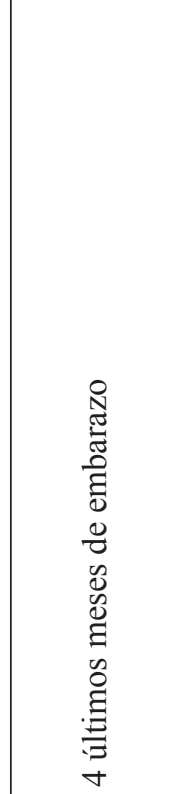 & 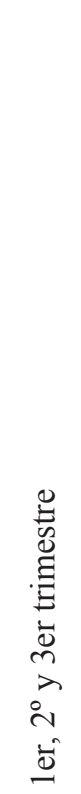 & 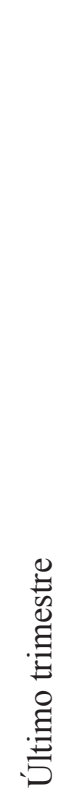 & 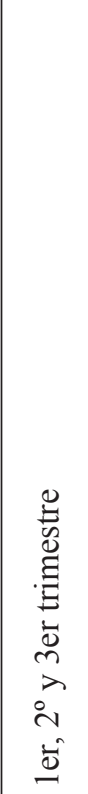 & 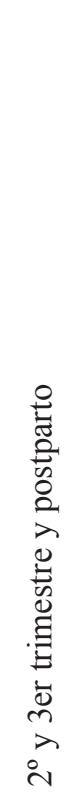 \\
\hline 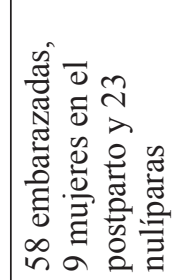 & $\tilde{n}$ & 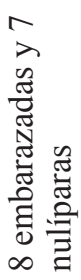 & $\stackrel{\curvearrowleft}{\sim}$ & $\underline{-}$ \\
\hline$\stackrel{ }{\stackrel{\sim}{\circ}}$ & $\stackrel{\infty}{\stackrel{\sim}{\sim}}$ & $\stackrel{\infty}{\stackrel{\infty}{\sim}}$ & $\stackrel{\infty}{\stackrel{\infty}{\sim}}$ & $\stackrel{\infty}{\stackrel{\infty}{\sim}}$ \\
\hline 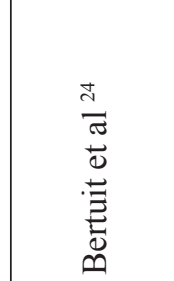 & 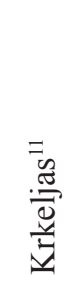 & 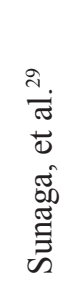 & 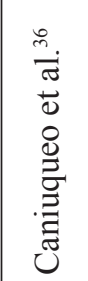 & 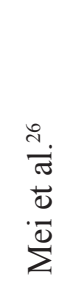 \\
\hline
\end{tabular}




\section{Discusión}

Los resultados de los estudios experimentales sobre la cinemática, la cinética y variables espaciotemporales son consistentes y confirman los hallazgos entre sí. En los últimos 5 años los estudios han sido capaces de evidenciar los cambios en la cinética que parecía aún sin definir completamente. En gran medida debido al desarrollo tecnológico que se ha producido para el análisis de la marcha.

La mayoría de los autores asocian estos cambios a un adaptación necesaria para asegurar una estabilidad en la marcha ${ }^{6,12,15}$ $20,35,37$ y es la causa que parece más razonable ya que algunas de estas variaciones dentro del ciclo de marcha se han observado en otras poblaciones ${ }^{38}$.

Las mujeres embarazadas prefieren caminar a una velocidad menor, que permite pasar más tiempo en doble apoyo y menor en apoyo unipodal en comparación con su patrón habitual. Así como realizar cambios espaciotemporales que también mejoran la estabilidad, como la disminución de longitud de paso y aumento de la anchura del paso, y el resto de cambios espaciotemporales ${ }^{39}$. Dichos cambios aumentan a medida que el embarazo avanza ${ }^{15}$.

Debido al aumento del abdomen, durante el embarazo hay desplazamiento del centro de gravedad progresivo y constante. Para intentar mejorar la estabilidad perdida la columna lumbar se adapta cuvándose en hiperlordosis, y que a medida que avanza el embarazo $\mathrm{y}$ el volumen abdominal aumenta pierde capacidad de adaptación ${ }^{35}$. Por ello, se produce consecutivamente la adaptación de la columna con una traslación lateral de las vértebras cervical $7^{\circ}(\mathrm{C} 7)$ y lumbar $4^{\circ}(\mathrm{L} 4)$, mayor en el último trimestre (asociada al aumento de la anchura del paso $)^{22}$, como mecanismo para centrar el centro de gravedad una vez que la hiperlordosis no deja realizar dicha función a la columna lumbar.

El miembro inferior también se adapta durante el embarazo ${ }^{40,38}$. Se ha observado que la cinemática de la marcha disminuye especialmente la extensión de cadera y la adducción y la plantar flexión del tobillo durante la marcha en el embarazo, sobre todo al final del embarazo. ${ }^{15}$ En el pie, se observa que disminuye el arco plantar significativamente en el primer trimestre ${ }^{40}$.

Estudios comparativos entre embarazadas y carga simulada del feto en mujeres nulíparas observan los mismos cambios en el patrón de la marcha. Lo que confirma que el aumento de peso sobre el abdomen es la principal causa del cambio en la marcha, por encima del resto de factores que afectan musculoesqueléticamente como la relaxina ${ }^{23}$

Esta pérdida del control del centro de gravedad tiene su repercusión en la línea que describe el centro de presiones durante la marcha. Su aumento significativo de desviación en el segundo y tercer trimestre del embarazo podría ser un indicador de una estabilidad reducida de la marcha y un aumento del riesgo de caída ${ }^{17,22}$, particularmente en la fase media de apoyo ${ }^{26}$. Esta desviación interna en fase final de contacto de talón e inicio de fase media de apoyo puede ser la causa de que se observe clínicamente un pie con arco plantar disminuido a las embarazadas ${ }^{40}$. Este hecho, unido a que presiones plantares aumentan y se redistribuyen a medida que aumenta el peso corporal en el embarazo $^{37}$, son dos posibles factores causales de las molestias en el mediopie de la embarazada, inclusive en mujeres sanas ${ }^{38}$

Como recomendaciones generales en esta población es importante pautar estiramientos de: gemelo, abductores de cadera, glúteo, isquiotibiales, y musculatura paravertebral lumbar, ya que aumentan los momentos de fuerzas que el pie debe generar para desplazarse 12,30. Así como movimientos de retroversión pélvica por el aumento de la lordosis ${ }^{35}$

$\mathrm{Se}$ debe recomendar fortalecer la musculatura del miembro inferior, ya que en estudios experimentales entre embarazadas que sufrieron alguna caída se observó que presentaban menor fuerza de impulso durante la marcha ${ }^{22}$

Son necesarias mayores investigaciones para saber los tratamientos ortopodológicos más adecuados en esta población en función de cada caso. Pero con el conocimiento actual podemos inferir que el uso de ortesis plantares puede ayudar a mejorar la estabilidad de la marcha, ya que se ha demostrado que las ortesis plantares ofrecen un efecto propioceptivo importante ya sólo con el cambio de textura sin tratamiento ortesico ${ }^{41}$.

Personas con patología relacionada con la laxitud ligamentosa previa al embarazo empeorarán en esta etapa debido al efecto de la relaxina. Como sucede en el caso del pie plano flexible. Se añadirán a las recomendaciones generales tratamiento ortopédico como soporte o sostén preventivo. Siempre teniendo en cuenta que la laxitud continua 6 meses 
posteriores al parto 9 . Del mismo modo son necesarios mayores estudios sobre tratamientos y medidas de prevención podológica para pies con patología previa.

\section{Conclusiones}

Se evidencian experimentalmente que existen cambios biomecánicos en la cinética y cinemática de la marcha durante el embarazo en miembro inferior, pie y tronco superior e inferior. Así como alteraciones las variables espaciotemporales, y estabilidad en la marcha. Sin embargo, aún son necesarios más estudios longitudinales para observar cambios desde el primer trimestre y su evolución y el estudio de los posibles tratamientos ortopédicos que pudieran ayudar a esta población en su marcha.

\section{Bilbiografía.}

1. Weir, Z. et al. Physical activity in pregnancy: A qualitative study of the beliefs of overweight and obese pregnant women. BMC Pregnancy Childbirth (2010). doi:10.1186/1471-2393-10-18

2. Rodacki, C. L., Fowler, N. E., Rodacki, A. L. \& Birch, K. Stature Loss and Recovery in Pregnant Women With and Without Low Back Pain. Arch Phys Med Rehabil 84, (2003).

3. Whitcome, K. K., Shapiro, L. J. \& Lieberman, D. E. Fetal load and the evolution of lumbar lordosis in bipedal hominins. Nature (2007). doi:10.1038/nature06342

4. Rasmussen, K. M., Catalano, P. M. \& Yaktine, A. L. New guidelines for weight gain during pregnancy: what obstetrician/gynecologists should know. Curr. Opin. Obstet. Gynecol. (2009). doi:10.1097/ GCO.0b013e $328332 \mathrm{~d} 24 \mathrm{e}$

5. Heslehurst, N. et al. The impact of maternal BMI status on pregnancy outcomes with immediate shortterm obstetric resource implications: A meta-analysis. Obesity Reviews (2008). doi:10.1111/j.1467789X.2008.00511.x

6. Branco, M., Santos-Rocha, R. \& Vieira, F. Biomechanics of Gait during Pregnancy. doi: $10.1155 / 2014 / 527940$

7. Calungueri, M., Bird, H. \& Wrigth, V. "Changes in jointlaxity occurring during pregnancy,". Ann. ofthe Rheum. Dis. 41, 126-128 (1982).

8. Schauberger, C. W. et al. Peripheral joint laxity increases in pregnancy but does not correlate with serum relaxin levels. Am. J. Obstet. Gynecol. (1996). doi:10.1016/S0002-9378(96)70447-7

9. Opala-Berdzik, A., Błaszczyk, J. W., Świder, D. \& Cieślińska-Świder, J. Trunk forward flexion mobility in reference to postural sway in women after delivery: A prospective longitudinal comparison between early pregnancy and 2- and 6-month postpartum follow-ups. Clin. Biomech. (2018). doi:10.1016/j. clinbiomech.2018.05.009

10. Whitcome, K. K., Shapiro, L. J. \& Lieberman, D. E. Fetal load and the evolution of lumbar lordosis in bipedal hominins. Nature 450, 1075-1078 (2007).

11. Krkeljas, Z. Changes in gait and posture as factors of dynamic stability during walking in pregnancy. Hum. Mov. Sci. 58, 315-320 (2018).

12. Foti, T., Davids, J. R. \& Bagley, A. A biomechanical analysis of gait during pregnancy. J. Bone Joint Surg. Am. 82, 625-32 (2000).

13. Fries, E. C. \& Hellebrandt, F. A. The influence of pregnancy on the location of the center of gravity, postural stability, and body alignment. Am. J. Obstet. Gynecol. 46, 374-380 (1943).

14. Sociedad Peruana de Obstetricia y Ginecología, J. E. Revista Peruana de ginecología y obstetricia. Rev. Peru. Ginecol. y Obstet. 63, 593-598 (2017).

15. Błaszczyk, J. W., Opala-Berdzik, A. \& Plewa, M. Adaptive changes in spatiotemporal gait characteristics in women during pregnancy. Gait Posture 43, 160-164 (2016).

16. Branco, M., Santos-Rocha, R., Aguiar, L., Vieira, F. \& Veloso, A. Kinematic Analysis of Gait in the Second and Third Trimesters of Pregnancy. J. Pregnancy 2013, 1-9 (2013).

17. Forczek, W., Ivanenko, Y. P., Bielatowicz, J. \& Wacławik, K. Gait assessment of the expectant mothers - Systematic review. Gait Posture 62, 7-19 (2018).

18. Hagan, L. \& Wong, C. K. Gait in pregnant women: spinal and lower extremity changes from pre- to postpartum. J. Womens. Health Phys. Therap. (2010). doi:10.1097/JWH.0b013e3181e8fd4d

19. Carpes, F., Griebeler, D., Kleinpaul, J., Mann, L. \& Mota, C. "Women able-bodied gait kinematics during and post preg- nancy period,". Rev. Bras. Biomec 'anica 9, 16 (2008).

20. Lymbery, J. \& Gilleard, W. The stance phase of walking during late pregnancy: temporospatial and ground reaction force variables. J. Am. Podiatr. Med. Assoc. (2005). 
21. Gilleard, W. L. Trunk motion and gait characteristics of pregnant women when walking: Report of a longitudinal study with a control group. BMC Pregnancy Childbirth (2013). doi:10.1186/1471-239313-71

22. McCrory, J. L., Chambers, A. J., Daftary, A. \& Redfern, M. S. The pregnant "waddle": An evaluation of torso kinematics in pregnancy. J. Biomech. 47, 2964-2968 (2014).

23. Aguiar, L. et al. Comparison between overweight due to pregnancy and due to added weight to simulate body mass distribution in pregnancy. Gait Posture 42, 511-517 (2015).

24. Bertuit, J., Leyh, C., Rooze, M. \& Feipe, V. Pregnancy-related changes in center of pressure during gait. Acta Bioeng. Biomech. 19, 95-102 (2017).

25. Hagan, L. \& Wong, C. K. Gait in Pregnant Women. J. Women's Heal. Phys. Ther. (2010). doi:10.1097/ JWH.0b013e3181e8fd4d

26. Mei, Q., Gu, Y. \& Fernandez, J. Alterations of Pregnant Gait during Pregnancy and Post-Partum. Sci. Rep. 8, 2217 (2018).

27. Vilador Pericé, A. Significado de la postura y de la marcha humana: (Teología, Antropología, Patología). (Complutense, 1996).

28. Sekiya, N. \& Yamazaki, H. Biomechanics and motor control of normal young adults performing a wheelchair wheelie balance task. Percept. Mot. Skills 110, 825-39 (2010).

29. Sunaga, Y., Kanemura, N., Anan, M., Takahashi, M. \& Shinkoda, K. Estimation of inertial parameters of the lower trunk in pregnant Japanese women: A longitudinal comparative study and application to motion analysis. Appl. Ergon. 55, 173-182 (2016).

30. HUANG, T.-H., LIN, S.-C., HO, C.-S., YU, C.-Y. \& CHOU, Y.-L. THE GAIT ANALYSIS OF PREGNANT WOMEN. Biomed. Eng. Appl. Basis Commun. 14, 67-70 (2002).

31. Andrade, S. R. de S. et al. Avaliação da inicialização da marcha na gestante de baixo risco nos três trimestres gestacionais. Rev. Bras. Ginecol. e Obs. 34, 376-380 (2012).

32. J.M., F., P., G., F.E., K., B., A. \& J., B. Gait coordination in pregnancy: A study in a rural population in Africa. Science and Sports (2009). doi:10.1186/1754-6834-6-22

33. Forczek, W., Ivanenko, Y. P., Bielatowicz, J. \& Wacławik, K. Gait assessment of the expectant mothers - Systematic review. Gait Posture 62, 7-19 (2018).

34. Eldeeb, A. M., Hamada, H. A., Abdel-Aziem, A. A. \& Youssef, A. M. The relationship between trunk and pelvis kinematics during pregnancy trimesters. Acta Bioeng. Biomech. (2016). doi:10.5277/ABB00544-2016-03

35. Sawa, R. et al. Differences in trunk control between early and late pregnancy during gait. Gait Posture 42, 455-459 (2015).

36. Caniuqueo, A., Fernandes, J., Quiroz, G., Rivas, R. \& Vargas, A. C. Revista Peruana de Ginecología y Obstetricia 109.

37. Inanir, A., Cakmak, B., Hisim, Y. \& Demirturk, F. Evaluation of postural equilibrium and fall risk during pregnancy. Gait Posture 39, 1122-1125 (2014).

38. Cartier R., L. Caídas y alteraciones de la marcha en los adultos mayores. Rev. Med. Chil. 130, 332-337 (2002).

39. Branco, M., Santos-Rocha, R. \& Vieira, F. Biomechanics of gait during pregnancy. Scientific WorldJournal. 2014, 527940 (2014).

40. Ponnapula, P. \& Boberg, J. S. Lower Extremity Changes Experienced During Pregnancy. J. Foot Ankle Surg. 49, 452-458 (2010).

41. Hijmans, J. M., Geertzen, J. H. B., Dijkstra, P. U. \& Postema, K. A systematic review of the effects of shoes and other ankle or foot appliances on balance in older people and people with peripheral nervous system disorders. Gait and Posture (2007). doi:10.1016/j.gaitpost.2006.03.010. 